\title{
Full-field X-ray Imaging, a Workhorse Microscopy Beamline at NSLS II for Material Science Researches
}

\author{
Xianghui Xiao ${ }^{1}$, Mingyuan $\mathrm{Ge}^{1}$, Chong Yin $^{2}$, Feng Wang ${ }^{3}$, Lijie Zou ${ }^{4}, 5$, Fei Chen ${ }^{4}$, Yu-chen Karen \\ Chen-Wiegart ${ }^{1,5}$ and Wah-Keat Lee ${ }^{1 *}$ \\ 1. National Synchrotron Light Source II, Brookhaven National Laboratory, Upton, NY, USA. \\ 2. Ningbo Institute of Materials Technology \& Engineering, Ningbo, China. \\ 3. Sustainable Energy Technologies Department, Brookhaven National Laboratory, NY, USA. \\ 4. State Key Laboratory of Advanced Technology for Materials Synthesis and Processing, Wuhan \\ University of Technology, Wuhan, China. \\ 5. Department of Materials Science and Chemical Engineering, Stony Brook University, Stony Brook, \\ NY, USA. \\ * Corresponding author: wklee@bnl.gov
}

The 18-ID Full-field X-ray Imaging beamline (FXI) at National Synchrotron Light Source-II (NSLS-II) started operation since late 2017. This beamline is equipped with a transmission X-ray microscope (TXM), which operates in x-ray energy $4.5-15 \mathrm{keV}$ range. The current highest achievable spatial resolution is $30 \mathrm{~nm}$. The uniqueness of this beamline is on its high temporal resolution, which is currently 20 seconds for a complete tomography scan. The high spatial and high temporal resolutions of the TXM at FXI therefore facilitates both in situ/operando and high throughput material science researches.

Figure 1 shows a tomographic reconstruction of a Siemens star pattern. The gap between the bars in the middle of the pattern is $30 \mathrm{~nm}$. The gaps can be clearly resolved in the tomographic reconstruction image. Such high spatial resolution imaging capability together with the fast scan speed is ideal for imaging integrated circuit (IC) chips. High-resolution IC chip imaging is highly desired in semiconductor community. The results help not only to understand the structure-functionality relationships but also to detect malicious features. In recent IC chip imaging test, we demonstrated we can conduct a local tomography scan of $45 \times 38 \times 10 \mathrm{um}^{3}$ volume at $70 \mathrm{~nm}$ resolution in 45 seconds. By conducting such tomographic scans at raster positions we can map a large size chip in short time. For instance, a $1 \times 1 \mathrm{~mm}^{2}$ size chip can be scanned in less than 10 hours. Fast scan time with TXM at FXI also facilitates imaging transient dynamic processes. As demonstrated in Ref [1], dynamic process of Ag whisker growth on the surface of a $\mathrm{Cu}$ wire in a substitution reaction was measured at FXI. The chemical reaction $\mathrm{Cu}+\mathrm{Ag}^{+}=$ $\mathrm{Cu}^{+}+\mathrm{Ag}$ occurred when the $\mathrm{Cu}$ wire immersed in $\mathrm{AgNO}_{3}$ solution. The shapes and growth rate of $\mathrm{Ag}$ whiskers depend on $\mathrm{AgNO}_{3}$ concentration. Ag whiskers grow into long dendrites in high $\mathrm{AgNO}_{3}$ concentration case $(0.5 \mathrm{mM})$, while into short nano-whiskers in low concentration case $(0.2 \mathrm{mM})$. These differences are clearly visible in the in situ tomography results and consistent with the results in literature.

In many cases, we are interested in not only morphology of a sample but also certain elements' concentrations in samples. This can be done by conducting tomographic scans at two x-ray energies, one below and another above the absorption edge of the concerned element. The differences between two scans highlight the distribution of the concerned element. Figure 2 shows the tomographic reconstructions from a $\mathrm{Cu}_{25} \mathrm{Fe}_{45} \mathrm{Al}_{30}$ alloy analyzed at below and above $\mathrm{Cu} \mathrm{K}$-edge. Fe-rich and $\mathrm{Cu}$-rich regions can be accurately determined in this way. Such information is important in correlating its structures to its processing conditions, and consequently in designing material structures. Beyond element concentration measurements, we can scan a sample at multiple energy points across the concerned element absorption 
edge. Such full-field mode X-ray Absorption Near Edge Spectroscopy (XANES) enables quick chemical state heterogeneity measurements. Thanks to the high flux available at FXI, we can perform quick fullfield XANES measurements without trade-offs on number of x-ray energy points and exposure time. Typical 2D XANES measurements take 10 minutes and 3D XANES measurements take 2 hours. Figures 3 (a) and (b) shows the 2D valence maps of $\mathrm{Ni}$ in composite NMC particles. The chemical heterogeneity is well distinguished between the shell and core, indicating that $\mathrm{Ni}^{2+}$ is mainly distributed in the nearsurface region of the particles, while $\mathrm{Ni}^{3+}$ is highly concentrated in the core. Figure 3(c) shows a slice image of two NMC532 particles from their 3D XANES measurements at Ni K-edge. At fully charge state, $\mathrm{Ni}$ is oxidized to $\mathrm{Ni}^{3+}$ (green colored) in most regions except of certain regions close to the solid-electrolyte interfaces, where small amount of $\mathrm{Ni}$ is still in $\mathrm{Ni}^{2+}$ (red colored) due to side reactions. Results from such TXM studies provided important information that will help to improve high cycling stability in the Nirich layered oxides by chemical heterogeneity [2].

\section{References:}

[1] M Ge et al., Appl. Phys. Lett. 113 (2018), p. 083109.

[2] This research used resources at FXI beamline (18-ID) of the National Synchrotron Light Source-II, a U.S. Department of Energy (DOE) Office of Science User Facility operated for the DOE Office of Science by Brookhaven National Laboratory under Contract No. DE-SC0012704.

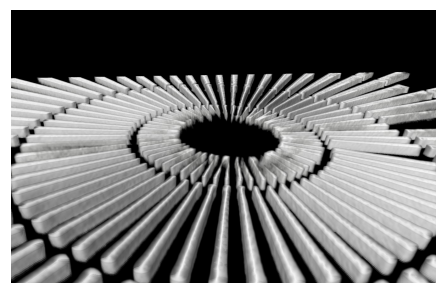

Figure 1. 3D structure of a Siemens star pattern. The gap between two finest bars in the middle is $30 \mathrm{~nm}$.

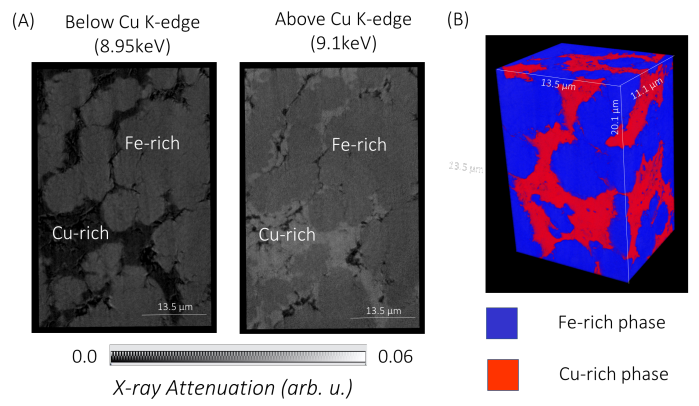

Figure 2. Analysis of $\mathrm{Cu}_{25} \mathrm{Fe}_{45} \mathrm{Al}_{30}$ alloy (A) pseudo cross-sections from below $\mathrm{Cu} \mathrm{K}$-edge and above $\mathrm{Cu}$ $\mathrm{K}$-edge data. (B) 3D volume rendering
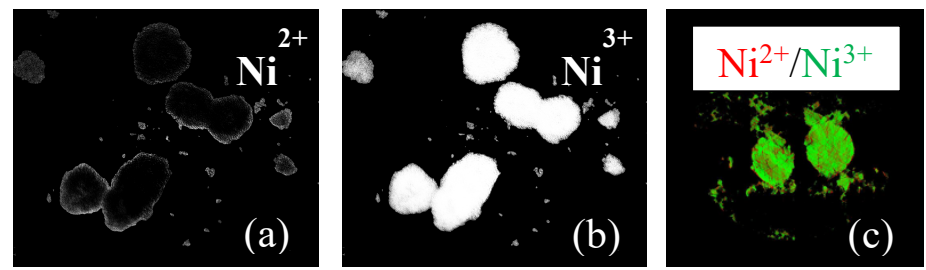

Figure 3. (a) $\mathrm{Ni}^{2+}$ and $\mathrm{Ni}^{3+}$ distributions in NMC electrode particles that have core-shell structure; (b) a 2D slice image of $\mathrm{Ni}^{2+}$ (red) and $\mathrm{Ni}^{3+}$ (green) distributions from a 3D XANES measurement. 